\title{
The Study of Integrated Information Platform Construction in Disaster Medicine Management
}

\author{
Sheng ZHONG \& Xin LIU \\ Business school, Sichuan University, Chengdu 610064, China
}

\begin{abstract}
This paper start from SARS and wenchuan earthquake to illustrate the importance of information security in the disaster, and find the development and problems when response to the rescue in China. Based on the information assurance problems existing in the activities of disaster medicine management, we come out of the necessity of integrated information platform construction in disaster medicine management. In addition, we try to construct the integrated information platform in disaster medicine management. Finally, in order to provide theoretical support for activities of medical Information assurance, we give some targeted suggestions to the construction of the integrated information platform and information assurance system in disaster medicine management.
\end{abstract}

KEYWORD: disaster medicine management; the integrated information platform; information assurance

\section{INTRODUCTION}

"Information security" (IA) is the concept that firstly put forward by the United States department of defense in the 1990s, which has been widely recognized after several times modified.[1] Information security described in this article is a series of measures, which is to meet the information need for all kinds of users in medical emergency management after disasters, including the establishment of the organization, information gathering, processing, communication, methods of the evaluation process, system platform support, etc. We try to fully exert the role of information in disaster medicine management for ensuring the smooth operation in the disaster medicine management.

Although China have response actively and made great progress in information security work during the SARS and the wenchuan earthquake, it still exposed problems during the sudden disaster, including the aspects of ideas, mechanism, institution, especially for the lag of the communication: (1) there is no uniform information platform, and the information acquisition still depend on each participators respectively (such as rescue teams, medical institutions, the fire department, etc.), leading to many information to be isolated islands. So the lack of a unified management is serious in the process. (2) Since there is no unified platform, information asymmetry and the long time of information acquisition will affect information release, even to the rescue activities.

As the Chinese disaster appears more frequently, the information becomes more particularly important in the relief, it can not only improve the effectiveness of disaster relief, but also will save lives in emergencies. Although this has attracted some attentions and some departments has built information platform in practice after "SARS" and wenchuan earthquake, it still have some problems. The fundamental problem is that all information do not standardize, different information cannot effectively share and exchange in one platform. This problem will only be solved after the construction of national information platform. However, we have many problems at the beginning of the construction. Firstly, we don't have a unified plan in information platform construction. Secondly, there is no specific unit or department to guarantee and take responsible for it. Finally, there is no capital and resources guaranteed to support the construction.

Although it is hard to construct the information platform in disaster medicine management, we might still push it to develop. There are five necessities as follows:

- A solid data support in disaster medicine

management

If we would have constructed the integrated information platform in disaster medicine management, we could improve the effectiveness of information collection, processing, maintenance and use. We could constantly complete information 
database of disaster prevention, disaster real-time information database, post-disaster reconstruction/assessment information database, etc., for providing a powerful data to decision command, disaster relief, energy security and resource scheduling in disaster medicine management.

- Strengthen the early-warning system in disaster medicine management

With information platform construction in disaster medicine management, we can have enough information to Omni-directionally and real-timely monitor anomalies, which equivalent to a permanent and complete system of early warning. Once there are signs of disaster information, the emergency department can make timely preparedness, announcing the public warning when necessary; other corresponding subjects will also be able to make preparations for disaster medical emergency in time, and avoid coughing off guard when disaster happened.

- Integration of emergency resource in disaster medicine management,

With information platform construction in disaster medicine management, we can complete overall architecture of management information system, construct information sharing platform in disaster medicine management which can adapt to a variety of tech, build the infrastructure components in different departments which can interconnect and sharing in the same standard. So we can break the regional segmentation, share with each other and improve the efficiency and accuracy of the disaster relief activities.

- Optimization of business process in disaster medicine management

With information platform construction in disaster medicine management, we could optimize business process in disaster medicine management for combining closely between the information system and activities in disaster medicine, shorting the response time of the disaster, providing a more scientific and rational solutions, and improving the effectiveness in disaster medicine management.

- Updating the contingency plans in disaster

medicine management

With information platform construction in disaster medicine management, we could improve the effectiveness of information collection, processing, maintenance and use. We could make all information sharing in the platform and constantly complete information database of disaster prevention, disaster real-time information database, post-disaster reconstruction/assessment information database, etc. Then we could use the helpful and real-time information to update contingency plans, in deeply, push the overall capability in disaster medicine management.

\section{LITERATURE REVIEW}

The amount of information and knowledge related to disaster management (for example, direct and indirect causes of disasters, and disaster avoidance or mitigation) increases exponentially. In the ultra complex setting of mega-disasters, decision-makers must process vast amounts of multidisciplinary, often poorly organized, disparate data and information and convert it into relevant and useable knowledge [2] Hays (1988), Cochrane(1991), Drabek and Hoetmer (1991), Gori (1991), Michaels (1992), Quarantelli (1993), IJMED (1993) Mileti (1999), Fothergill (2000), IFRCRCS (2005) and ISDR (2005) states that an increase in the circulation of disaster information does not always correspond with increased assimilation of new disaster knowledge.[3] McEntire (2002) claimed that information challenges and the lack of communication between the field and the operation centre during the March 2000 post disaster mission to address the ramifications of the Fort Worth (Texas, United States) tornado were due to inaccurate, incomplete or too much information, which caused delays in decision-making, as well as insufficient information in some cases $^{[4]}$. It is undeniable that contemporary information technologies (IT) have changed disaster communications. The application of computer networks, digital libraries, satellite communications, remote sensing, Geographic Information Systems (GIS) and decision support systems to emergency management; [4] Dash (1997), Fisher(1998), Quarantelli (2002), Kaiseret al. (2003), Thomas et al. (2003), ISDR (2005), Thomas Ertugay and Kreme claimed [3]. ICT plays an important role in DM, facilitating the process of information flow and coordination and enhancing disaster planning, mitigation and management. As the number of system users increases, it gets harder to ensure the adequacy of the information flow; [3] Sagun, Bouchlaghem and Anumba (2006); [4] In practice, several information systems have been implemented with just that goal and still, adequate system performance in disaster relief supply chains remains elusive (McEntire, 1999, 2002; Stephenson, 2005; Wise, 2006). [5]

Based on the "management" defined as "an organization behavior which makes resources to meet demand ", Dongwang comes out of some ideas about the disaster medicine management including connotation, theoretical framework, research methods and its relationship with other subjects; [6] Yige and Zhenhe analyze the problems existing in information collection of the emergency medical rescue, and they gives some advice of information classification based on features of emergency medical rescue[7]-[8]; In order to increase the medical information service work of army medical 
personnel, Ping Zheng and Shulian Luo make an analysis range from the current status of emergency medical informatics to the establishment and functions of emergency medical informatics system. Li Xu, Ran Zheng (2013) make an analysis about the characteristics of recovery force based on "four stages, a principle, a system", and also analyzes the process of information security, which provide troops theoretical foundation in information management of disaster relief.

Academically, there are already some systematically researches in the field of the characteristics of disaster information and the importance of information communication in emergency management system in global.

After the terrorist attacks on the world trade center, the United States started to prepare for early warning systems which send emergency information though telephones when major natural disasters or terrorist attack happened. FEMA and other website of emergency service, some folk groups, will also release and update the latest information on relief in real time. They have established information exchange and sharing between departments of federal and local governments, local and local. And state command center, the related research institutions and the national meteorological center has realized the exchange of information, which can get information at any time by the satellite. These measures ensure that warning message could access the public via a variety of means when major crisis occurs.

In 2005, many developed countries began to start the research of information system in medical rescue as well as the construction of sharing information platform of regional emergency medical rescue, and built the national medical rescue information network. All they have done has caused the sharing of scattered resources.

We have done lots of academic research on information collection and security in disaster relief in China. After "SARS" and Sichuan wenchuan earthquake, this field has attracted some attention in practice, but has not yet been built unified and standardized medical information security system. There is still no systematic study of integrated information platform construction in disaster medicine management in China. In order to fill the void, this paper will come up with some ideas and focus on in integrated Information Platform construction in disaster medicine management.

\section{THE INTEGRATED INFORMATION PLATFORM}

Firstly, we will construct the framework of integrated information platform construction in disaster medicine management (figure 1). Secondly, in order to maintain the platform work well, we need the operation management which is a continuously updated process (as the circle of platform maintenance, normal corporation management, operation management in disaster and platform update after disaster), as shown in figure 2.Thirdly, based on platform in figure 1, we try to make out how every core module construct one by one (information collection module, information analysis module, decision support module, information publish module). Finally, we construct information assurance system in disaster medicine management (Figure 4).

\subsection{The framework}

The integrated information platform in disaster medicine management affiliates to the national emergency information platform. And the author believes that countries should set up a professional team to build a national emergency information platform, as well as the integrated information platform in disaster medicine management as a part of a national emergency information platform.

As a unified carrier which convenient for flow of information, the information platform in disaster medicine management will mainly have access to various subjects which have connection with the rescue activities for sharing information and improving the effectiveness of work. Such a huge integrated information platform, so we can't use a single graphics to describe clearly. In order to illustrate this, the author set up figure 1 to describe the juche idea:

Firstly, we should have the information collection module at the periphery of the platform which accesses the platform. Secondly, there are another main four modules in the platform inside, following as the order including the modules of information input, information analysis, decision support and information publish. Then all these five modules form the main line. In addition, there are some subjects which accesses to each module which are not only the maintenance subjects, but also the main consuming bodies. Finally, the main line and all subjects of periphery form a loop.

It is worth mentioning that the nature of information itself is dynamic, continuously updated. The feedback of information flow it generate will become the new information recourse which put into the collection phase, so the information release is geared to the needs of the society as a whole, almost all the subjects involved in disaster rescue. So the integrated information platform in disaster medicine management forms a circular platform, and the peripheral platform needs corresponding maintenance module. 


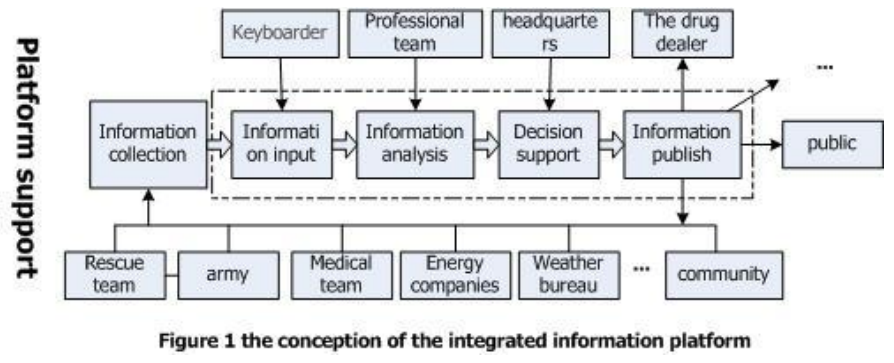

\subsection{The operations management}

After the construction of integrated information platform in disaster medicine management, we need the operation management which is a continuously updated process, as shown in figure 2 . There should be a support of platform maintenance to ensure the work of platform. Before the disaster happened, we need normal operation management, especially pay more attention to information collection, information input and early warning forecast. In addition, normal operation management will help people to be more familiar in how to use it, and improve the effectiveness and short the reaction time when the disaster occurs. During the period of disaster, the information platform start engine and all participants (subjects) who have connection with the main line could find the information they need. The activities including decision-making, rescue, protection, support and communication could carry out orderly and efficiently when the engine starts. In order to achieve high degree of coordination in rescue activities, we must require the support of sharing information. And the priority is to guarantee features of information, including comprehensiveness, timeliness, smooth and sharing. After disaster under control, we need to assess the operation of the platform and find out problems, timely updates to make sure that every process could be better when the next disaster occurs. After the updating, the platform starts the next round of maintenance, which forms a cycle of operation management process.

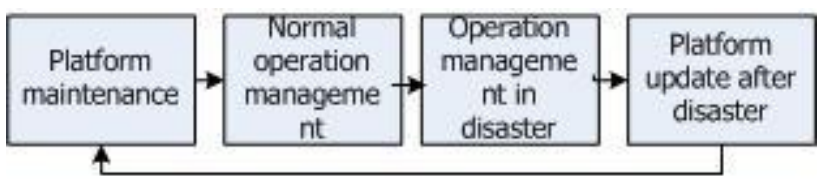

Figure 2 operation management of the integrated information platform

\subsection{The Core areas}

We have described integer conception and overall operations management of the integrated information platform in disaster medicine management (as shown in the previous part). Then we will give specific construction plan of five core modules, including information collection, information input, information analysis, decision support and information publish (as shown in figure 1). Before this, we must firstly go on the demand analysis and information classification.

\subsubsection{Information demand analysis and classification}

The whole process of disaster medicine management cannot go on wheels without the information. Before the disaster, we need ecological information including the geology, water quality, climate, forest coverage; we also need the social information such as population density, population structure, the main way of life and the local history of disaster; the basic information, including the number and size of local gym, clearing and school. When disaster occurs, we need instant message to support the rescue activities including the nature of disaster, scale, transmission speed, casualties, supply and demand situation of drinking water and food, the blood supply and demand, medicines, medical apparatus and instruments, clothing, tents, masks, supply and demand situation of disinfection products, number of houses collapsed, the pathway, electricity and communications repair, weather conditions; After the disaster under control, we need the after-calamity information including body land filling, disinfection and personnel placement, secondary disaster situation, the water pollution, pathways, the situation of electricity and communications blocking, ecological damage, direct economic loss of disaster.

Disaster medicine management can be divided into five activities which are decision-making, rescue, protection, support and communication activities, and each activity needs different information. The main subject of the decisionmaking activity is governments at all levels, and they always focus on the information about supply and demand situation of personnel and material to facilitate scheduling decisions. At the same time, they also extremely need all the information after a disaster to restore and update.

The main subjects of the rescue activity mainly include the army, medical institutions and volunteers. And their mission is to save lives, so they mainly need information about supplies and demand of patients and medicine. The main subjects of security activity are the community, the local big energy companies, blood Banks, large pharmaceutical medicines and medical equipment enterprises. In order to ensure the disaster relief activities, they always focus on the information about electricity and communications, blood supply and demand situation and the information of drugs and medical equipment. The main subjects of the support activity are enterprises, the public, charitable organizations and foreign governments, and they mainly provide the peripheral support for disaster relief, focusing on the information of supply and demand in drinking water, food, clothing, tents. The subjects of communication activities are mainly the 
media and the social public, and they need almost all aspects of information.

According to these information demand analysis, we can found that we need a very large amount of information in the process of the whole management. In the view of disaster medicine management, we divided all information into three categories.(1) The class A is directly related to medical information: the supply and demand information about local blood, medicines, medical equipment and appliance; disaster degree, casualties, the scale of the local medical institutions, the local infectious diseases and history information of sudden disaster relief, professional rescue team size, the supply and demand situation of water and food, speed and scope of the disaster and so on;(2) The class $\mathrm{B}$ is strongly associated with medical information: population density, the local culture and customs, water supply, pathways, disaster spread speed and scope, weather conditions, the ecological damage and water pollution after a disaster and so forth;(3) The class $\mathrm{C}$ is weekly related to medical information: ecological conditions, including local topography, geomorphology, geology, climate; the local people's living standard, population structure, houses collapsed and the information about direct economic losses and so on.

\subsubsection{Construction of information collection module and information input module}

In the concept of integrated information platform in disaster medicine management, information collection plays an important role in the circle. In the former part, we have done demand analysis. In order to complete the part of information collection, we introduce the subjects of information collection. Academy of social sciences, meteorology and the community are both collection sources for class B and C. Early warning and emergency government departments and the media are almost the information collection sources. All these subjects are required to access the integrated information platform as the peripheral collection module. Then the professional team in figure 1 could do a better information analysis after this series of information. collection, which provides information basis for decision support.

After information collection, we need professional personnel put all information into resource library of platform, just for later analysis and decision support.

\subsubsection{Construction of the decision support module}

After the information input, we need to analyze information systematically and unify standards to ensure the highly liquidity of the information in each link of the whole platform, so as to achieve sharing. At the same time, we should collate information again based on different need of different subjects, then shunt to the corresponding subjects for decision support. After collating information, we should distribute information reasonably to the corresponding module which facing their own subjects, eventually forming a front platform which helps different users directly get information they need in the website (the entry of the front platform).

As shown in figure 3 , the decision-making information sub platform in disaster medicine management is in the position of the center linked with communication the sub platform, and both covered all the disaster rescue activities. Under the coordination of information communication sub platform and the leadership of decision-making information sub platform, corresponding rescue information sub platform, ensuring information sub platform and support information sub platform can work well. The front platform located in the decision-making module in figure 1 , which also an application platform serving for users. Every subject can find their own position and corresponding entry to get the information they need, just click in and then they will find the information which related to them.

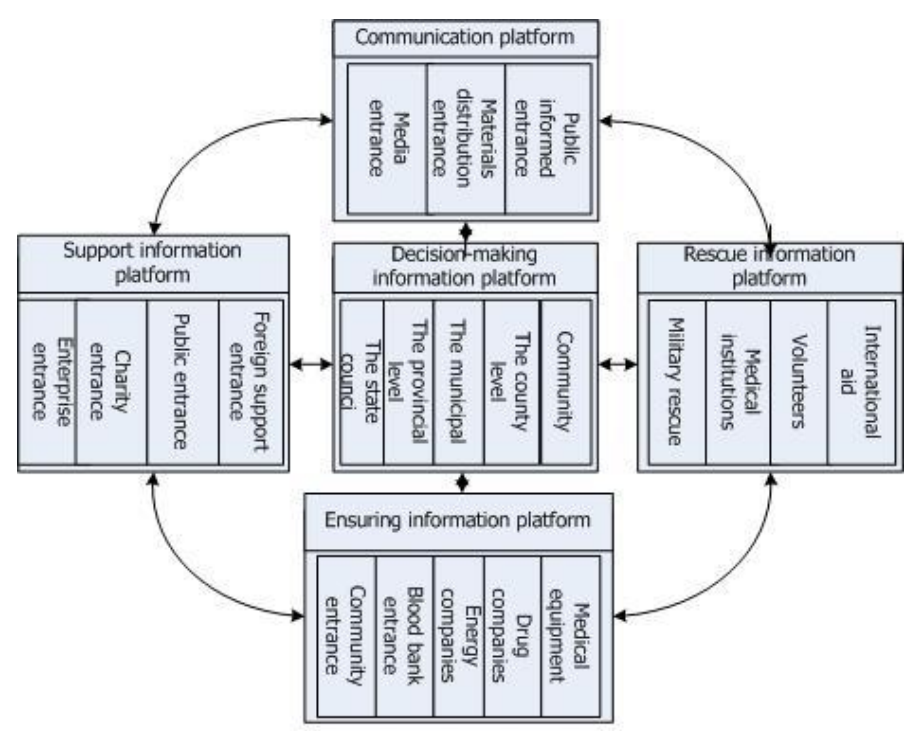

Figure 3 the user front-end platform of the integrated information platform

\subsubsection{Construction of information assurance system}

The integrated information platform construction in disaster medicine management must base on data support from the information assurance system. The author constructed an all-round support information system diagram in disaster medicine management with a time axis based on pre disaster, during disaster and post disaster, and a vertical axis based on the collection stage, the information processing stage and the information utilization stage. The diagram is shown in Figure 4:

In the information collection stage before the disaster, we should build the prevention information collection system, including some subsystems: Geographic information collection system, 
ecological information collection system, the social information collection system and history of disaster information collection system. Then we can get the disaster prevention information database for use. In the information collection stage during the disaster, we should build the disaster information collection system, including some subsystems: Decisionmaking information collection system, rescue information collection system, assurance information collection system, supporting information collection system and communication information collection system. Then we can get the disaster real-time information database for use. In the information collection stage after the disaster, we should build the after-calamity information collection system, including some subsystems: casualties' information collection system, the economic loss of information collection system, ecological damage information collection system and disaster result information collection system. Then we can get the disaster assessment information database for use.

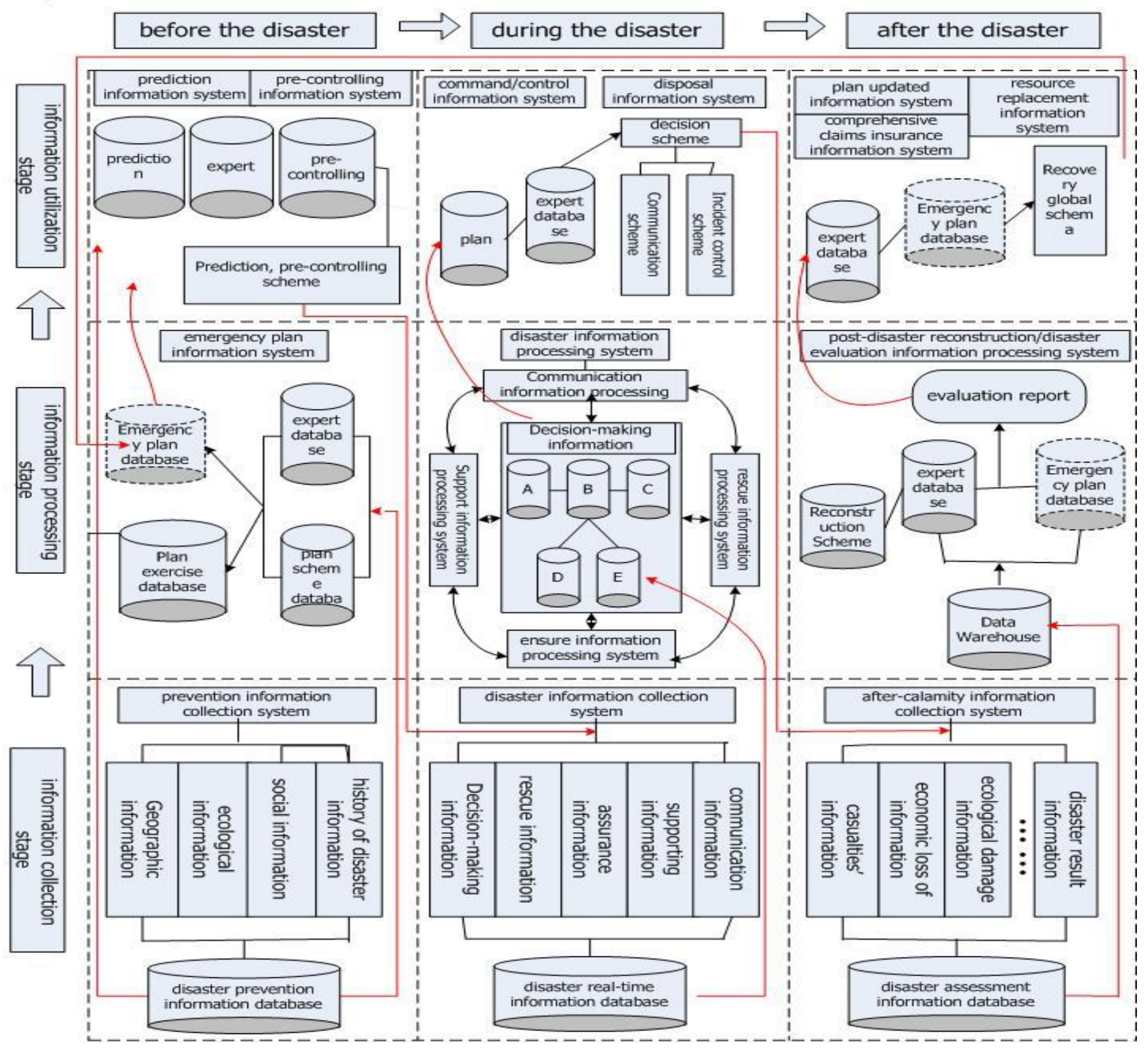

Figure 4 The information assurance system diagram

We have different information handling system in different period in the information processing stage. Before the disaster, we should build emergency plan information system; during the disaster, we should build the disaster information processing system; after the disaster, we should build post-disaster reconstruction/disaster evaluation information processing system.

In the information utilization stage before the disaster, we should build prediction information system and pre-controlling information system; during the disaster, we should build command/control information system and disposal 
information system; after the disaster under the control, we should build plan updated information system, resource replacement information system and comprehensive claims insurance information system.

Tips: in the figure $6, \mathrm{~A}$ is decision-making model database, $\mathrm{B}$ is expert database, $\mathrm{C}$ is decision-making plan database, $\mathrm{D}$ is case database, $\mathrm{E}$ is data warehouse.

\section{SUGGESTIONS}

We have discussed the overall framework of integrated information platform construction in disaster medicine management. However, if we want to give full play to information security role of platform in real relief work, we have to emphasize the following points:

\section{- To strengthen the construction of data}

standardization and data processing capacity

The design of integrated information platform in disaster medicine management should be based on the general principles, linked with system module to ensure the consistency and compatibility of platform, to ensure the information data communication smoothly. In order to reach the goal of sharing information and compatibility, the priority is to solve the problem of data standardization. Standardized processing is the only thing to improve liquidity of data between the various functional modules or sub platforms. After data standardization, we must strengthen the filtration function (data processing, data analysis, classification). And the filtration function is the only way to ensure the efficient operation of the entire information security system, and then play the role of integrated information platform.

\section{- Strengthen the response mechanism and}

coordination function of the system

For Emergency response, the speed is the key. The meaning of integrated information platform construction is to solve the question of coordination in complex situation and to move in tandem. In order to give play to the role of decision support, we needs making detailed and exhaustive emergency information security plan. And the relevant departments should strengthen the response mechanism of the whole system, especially paying more attention on important time and location. Once disaster appears, we could ensure that the disaster medical information security task can be completed immediately with the fastest speed. We should strengthen the coordination function between various subsystems. It is not a simple fusion of various modules but a coordinating organic whole.

- Paying attention to reciprocal interaction of platform
The integrated information platform construction in disaster medicine management is to satisfy information need of subjects and ensure the effectiveness of disaster relief activities. In order to meet the updating of information platform, the updating information is provided from every subject. Therefore, the information release platform is facing to all subjects who have access to the platform. On the one hand, it provides timely information for disaster relief activities. On the other hand, we also achieve the purpose of information updating by subjects information collection, which formed a twoway interactive platform. In order to be both coordination and flexibility, we must pay more attention to the two-way interactive due to disaster information changing fast.

- To ensure the scalability of the system

The integrated information platform construction in disaster medicine management does not do once and for ever, it needs to be constantly updated and expanded time and time again so as to ensure the platform continues to play a role. With the constantly improvement of the information technology and the emergency medical rescue teams, fixed disaster medical information security system can't satisfy the needs of future disaster medicine management. Therefore, information security system construction in disaster medicine management should be extended based on the business development of disaster activity to ensure the scalability of the system.

- To do a good job of risk management of the

platform

The integrated information platform in disaster medicine management is a complex giant system that involves various subjects. In order to ensure information being authentic, reliable, we must to do a good job of risk management of the platform. On the one hand, related subjects have their own access permissions and update for the information collection, so that any information has corresponding principal, who ensure the authenticity and reliability of their information. On the other hand, each subject can only get the information needed for the emergency work, to ensure information security and confidentiality of the platform.

- overall planning and construction Step by step

The integrated information platform in disaster medicine management is a large and complex system, which have a large of the subjects and a great amount of data. At the same time, it involved in different subsystem including communications, hardware, software, which means it can't construct overnight. So it is necessary to highlight key and strengthen the demonstration in the process of platform construction. It can firstly use the army's existing emergency platform to gradually expand outward. Integrated information platform of Army 
can play an exemplary role which lead to construct the platform in the national scope.

\section{REFERENCES}

[1] Xiang shenchang, Yicai and Zhao zheliang. Information Security Engineering. COMPUTER ENGINEERING AND SCIENCE, 2002, 24(2).

[2] Dag K.J.E. von Lubitz, James E.Beakley and Frederic Patricelli. All hazards approach to disaster management: the role of information and knowledge management, Boyd's OODA Loop, and network-centricity. Disasters. Dec2008, Vol. 32 Issue 4, p561-585. 25p. 3 Diagrams, 2 Charts.

[3] Marincioni, Fausto.Information technologies and the sharing of disaster knowledge: the critical role of professional culture. Disasters. Dec2007, Vol. 31 Issue 4, p459-476. 18p. 1 Diagram, 4 Charts.

[4] Aysu Sagun, Dino Bouchlaghem and Chimay J. Anumba. A scenario-based study on information flow and collaboration patterns in disaster management. Disasters. Apr2009, Vol. 33 Issue 2, p214-238. 25p. 7 Diagrams, 5 Charts, 1 Map.

[5] Day, Jamison M. Junglas, Iris. Silva, Leiser. Journal of the Association for Information Systems. Aug2009, Vol. 10 Issue 8, p637-660. 24p. 2 Diagrams, 3 Charts.

[6] Dongwang. Thinking on constructing the discipline of Disaster Medicine Management. CHINESE HEALTH SERVICE MANAGEMENT, 2011, 1:9-10.
[7] The problems and countermeasure existing in the emergency medical rescue information acquisition People's Military Surgeon, 2012, 55(3):204-205.

[8] Wang qingyang, Yige and Zhenhe. The characteristics and classification of emergency medical rescue information. People's Military Surgeon, 2013, 55(3): 200-201.

[9] Pingzhen, Luo shulian. The present situation of research, the content and Emergency in information about medical informatics. HAINAN MEDICAL JOURNAL, 2010, 21(16): 125-126.

[10] Lixu,Ranzhen and Huang zhaohui. The thinking of security features and process in disaster emergency medical rescue. Southwest national medical defense, 2013, 23(5): 561-563.

[11] Zhang yanling. Ponderation on the strategy of medical rescue after wenchuan earthquake. MEDICAL JOURNAL OF CHINESE PEOPLE'S LIBERATION ARMY, 2009, 34(1):1-6

[12] Lieahen, Bopei and Cheng mingfu The concept and features of emergency medical rescue organizations. People's Military Surgeon, 2010, 53(12):958-960.

[13] Zhang yanping. Information resources integration research in China. INFORMATION RESEARCH, 2008, 124(2): 47-49.

[14] Chaowang, Yige,Z hongzhen, Huang Tianna and Weipu, The function of emergency medical rescue information system. People's Military Surgeon, 2012, 55(3):197-199.

[15] Yang cuibin. Analysis and design of provincial emergency management information system — put shandong province as an example. Department of business administration in shandong university, 2009. 